\title{
ON THE KERNEL OF THE GASSNER REPRESENTATION
}

\author{
KEVIN P. KNUDSON
}

\begin{abstract}
We study the Gassner representation of the pure braid group $P_{n}$ by considering its restriction to a free subgroup $F$. The kernel of the restriction is shown to lie in the subgroup $\left[\Gamma^{3} F, \Gamma^{2} F\right]$, sharpening a result of Lipschutz.
\end{abstract}

\section{INTRODUCTION}

Denote by $G_{n}: P_{n} \rightarrow G L_{n}\left(\mathbb{Z}\left[t_{1}^{ \pm 1}, \ldots, t_{n}^{ \pm 1}\right]\right)$ the unreduced Gassner representation of the pure braid group $P_{n}$ (a formula is given in Section 2 below). The faithfulness of $G_{n}$ for $n \geq 4$ is a long-standing open question. In this note, we investigate this by considering the restriction of $G_{n}$ to a certain free subgroup $F_{n-1}$ of $P_{n}$ :

$$
g_{n}: F_{n-1} \longrightarrow G L_{n}\left(\mathbb{Z}\left[t_{1}^{ \pm 1}, \ldots, t_{n}^{ \pm 1}\right]\right)
$$

The faithfulness of $G_{n}$ would follow from that of $g_{n}$ (see Proposition 2.1 below, or [3] for a more general result).

For a group $H$, denote by $\Gamma^{\bullet} H$ the lower central series of $H$. The main result of this paper is the following.

Theorem 3.4. The kernel of $g_{n}$ lies in the subgroup $\left[\Gamma^{3} F_{n-1}, \Gamma^{2} F_{n-1}\right]$.

This is proved by passing to the graded quotients associated to the lower central series of $F_{n-1}$ and the filtration of $G L_{n}\left(\mathbb{Z}\left[t_{1}^{ \pm 1}, \ldots, t_{n}^{ \pm 1}\right]\right)$ by powers of the augmentation ideal $J=\operatorname{ker}\left\{\mathbb{Z}\left[t_{1}^{ \pm 1}, \ldots, t_{n}^{ \pm 1}\right] \stackrel{t_{i} \mapsto 1}{\longrightarrow} \mathbb{Z}\right\}$. This allows us to show that the kernel of $g_{n}$ lies in $\Gamma^{5} F_{n-1}$. In [8], S. Lipschutz proved that the kernel of $g_{n}$ lies in $\left[\Gamma^{2} F_{n-1}, \Gamma^{2} F_{n-1}\right]$ using different techniques (see also [1] for another proof). These two facts together allow us to prove Theorem 3.4

We also show (Theorem 3.5) that the intersection of the kernel of $g_{n}$ with $\Gamma^{s} F_{n-1}$ lies in the subgroup $\left[\Gamma^{s-2} F_{n-1}, \Gamma^{2} F_{n-1}\right] \cdot \Gamma^{s+1} F_{n-1}$.

Acknowledgements. I thank Fred Cohen for suggesting this question to me and for many useful conversations. I am also grateful to an anonymous referee for many helpful comments.

\section{Preliminaries and Notation}

2.1. The Gassner representation. Denote by $A_{r s}, 1 \leq r<s \leq n$, the generators of $P_{n}$. The (unreduced) Gassner representation is the homomorphism $G_{n}: P_{n} \rightarrow$ $G L_{n}\left(\mathbb{Z}\left[t_{1}^{ \pm 1}, \ldots, t_{n}^{ \pm 1}\right]\right)$ given by the formula:

Date: November 1, 2004.

1991 Mathematics Subject Classification. 20F36.

Partially supported by NSF grant no. DMS-0242906 and by ORAU. 


$$
G_{n}\left(A_{r s}\right)=\left(\begin{array}{ccccc}
I_{r-1} & 0 & 0 & 0 & 0 \\
0 & 1-t_{r}+t_{r} t_{s} & 0 & t_{r}\left(1-t_{r}\right) & 0 \\
0 & \vec{u} & I_{s-r-1} & \vec{v} & 0 \\
0 & 1-t_{s} & 0 & t_{r} & 0 \\
0 & 0 & 0 & 0 & I_{n-s}
\end{array}\right)
$$

where

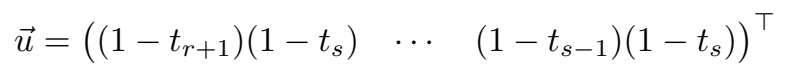

and

$$
\vec{v}=\left(\begin{array}{lll}
\left.\left(1-t_{r+1}\right)\left(t_{r}-1\right) \quad \cdots \quad\left(1-t_{s-1}\right)\left(t_{r}-1\right)\right)^{\top} & \cdots
\end{array}\right.
$$

and $I_{k}$ denotes the $k \times k$ identity matrix. This representation is reducible to an $(n-1)$-dimensional representation, but the resulting formula is more complicated.

2.2. The free subgroup. Denote by $F_{n-1}$ the free subgroup of $P_{n}$ obtained by deleting the last string; this subgroup has generators $A_{1 n}, A_{2 n}, \ldots, A_{n-1, n}$. Moreover, we have a split short exact sequence

$$
1 \longrightarrow F_{n-1} \longrightarrow P_{n} \longrightarrow P_{n-1} \longrightarrow 1
$$

so that $P_{n}$ is the semidirect product of $P_{n-1}$ and $F_{n-1}$. Also, the following diagram commutes (2], p. 138):

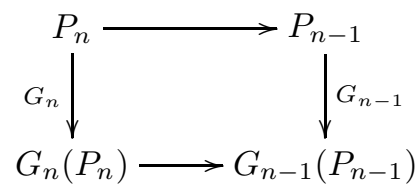

where the lower horizontal map is given by setting $t_{n}=1$ and deleting the $n$th row and column.

Denote by $\Gamma^{\bullet} F_{n-1}$ the lower central series of $F_{n-1}$ and for each $i$, consider the free abelian group

$$
\Gamma^{i} F_{n-1} / \Gamma^{i+1} F_{n-1}
$$

We shall need an explicit basis of each $\Gamma^{i} F_{n-1} / \Gamma^{i+1} F_{n-1}$; this is given by the set of basic commutators of weight $i$. These are defined as follows. Denote by $x_{j}$ the image of $A_{j n}$ in $F_{n-1} / \Gamma^{2} F_{n-1}$. Then the $x_{j}$ are the basic commutators of weight one (denote this by $w\left(x_{j}\right)=1$ ) and having defined the basic commutators of weight less than $i$, the basic commutators of weight $i$ are the various $\left[c_{u}, c_{v}\right]$ where

(1) $c_{u}$ and $c_{v}$ are basic with $w\left(c_{u}\right)+w\left(c_{v}\right)=i$, and

(2) $c_{u}>c_{v}$ and if $c_{u}=\left[c_{a}, c_{b}\right]$, then $c_{v} \geq c_{b}$.

The commutators are ordered as follows. Those of weight $i$ follow those of weight less than $i$ and are ordered arbitrarily with respect to each other. A proof that the basic commutators of weight $i$ form a basis of $\Gamma^{i} F_{n-1} / \Gamma^{i+1} F_{n-1}$ may be found in 4], p. 175.

Denote by $g_{n}$ the restriction of $G_{n}$ to $F_{n-1}$ and set $X_{n}=g_{n}\left(F_{n-1}\right)$.

\section{Proposition 2.1.}

$$
\begin{aligned}
G_{n} \text { is faithful } \Leftrightarrow & g_{n} \text { is faithful } \\
\Leftrightarrow & \text { the map } \Gamma^{i} F_{n-1} / \Gamma^{i+1} F_{n-1} \rightarrow \Gamma^{i} X_{n} / \Gamma^{i+1} X_{n} \\
& \text { is injective for each } i \geq 1 .
\end{aligned}
$$


Proof. A proof of the first equivalence may be found in, for example, 3. The second equivalence is an easy exercise about free groups and is left to the reader.

2.3. The congruence subgroup. Denote the subgroup of $G L_{n}\left(\mathbb{Z}\left[t_{1}^{ \pm 1}, \ldots, t_{n}^{ \pm 1}\right]\right)$ consisting of those matrices $A$ with $A \equiv I_{n}$ modulo $\left(t_{1}-1, t_{2}-1, \ldots, t_{n}-1\right)$ by $K_{n}$ (i.e., $K_{n}=G L_{n}(R, J)$ for $R=\mathbb{Z}\left[t_{1}^{ \pm 1}, \ldots, t_{n}^{ \pm 1}\right]$ and $J$ the augmentation ideal). Note that the image of $P_{n}$ under $G_{n}$ lies in $K_{n}$. The group $K_{n}$ is filtered by powers of $J$ :

$$
K_{n}^{i}=\left\{A \in K_{n}: A \equiv I_{n} \bmod J^{i}\right\} .
$$

This is a central series: $\left[K_{n}^{i}, K_{n}^{j}\right] \subseteq K_{n}^{i+j}$.

Consider the graded quotients $K_{n}^{i} / K_{n}^{i+1}$. Note that $\Gamma^{i} K_{n} \subseteq K_{n}^{i}$, but it is probably not true that $K_{n}^{\bullet}$ is the lower central series (for $R=\mathbb{Z}\left[t, t^{-1}\right]$, the corresponding group $K_{n}$ has $K_{n}^{i} / \Gamma^{i} K_{n}$ a torsion group for $n \geq 4$ [7]). Consider the induced map

$$
\Phi^{i}: \Gamma^{i} F_{n-1} / \Gamma^{i+1} F_{n-1} \longrightarrow K_{n}^{i} / K_{n}^{i+1} \text {. }
$$

Then by Proposition 2.1] we have the following:

$$
G_{n} \text { is injective if } \Phi^{i} \text { is injective for all } i \geq 1 \text {. }
$$
$k=5$.

We show in Section 3 that $\Phi^{k}$ is injective for $k \leq 4$, but that injectivity fails for 2.4. Structure of $K_{n}^{i} / K_{n}^{i+1}$. Given $A \in K_{n}^{i}$, we may write

$$
A \equiv I_{n}+\sum_{1 \leq \ell_{1} \leq \cdots \leq \ell_{i} \leq n}\left(t_{\ell_{1}}-1\right) \cdots\left(t_{\ell_{i}}-1\right) A_{\ell_{1}, \ldots, \ell_{i}} \bmod J^{i+1},
$$

where $A_{\ell_{1}, \ldots, \ell_{i}} \in M_{n}(\mathbb{Z})$. Define

$$
\pi_{i}: K_{n}^{i} \longrightarrow \bigoplus_{1 \leq \ell_{1} \leq \cdots \leq \ell_{i} \leq n} M_{n}(\mathbb{Z})
$$

by

$$
\pi_{i}(A)=\left(A_{\ell_{1}, \ldots, \ell_{i}}\right)_{1 \leq \ell_{1} \leq \cdots \leq \ell_{i} \leq n} .
$$

$\pi_{i}$ is clearly a homomorphism and $\operatorname{ker} \pi_{i}=K_{n}^{i+1}$.

Denote by $e_{\ell m}(a)$ the matrix having $a$ in the $\ell, m$ position and zeroes elsewhere. Note that $\pi_{1}$ is surjective:

$$
\pi_{1}\left(I_{n}+e_{\ell m}\left(t_{j}-1\right)\right)=\left(0, \ldots, 0, e_{\ell m}(1), 0, \ldots, 0\right)
$$

where $e_{\ell m}(1)$ appears in the summand corresponding to $\left(t_{j}-1\right)$. Note that this works for $\ell=m$ as $1+\left(t_{j}-1\right)=t_{j}$ is a unit in $\mathbb{Z}\left[t_{1}^{ \pm 1}, \ldots, t_{n}^{ \pm 1}\right]$. For $i>1$, the image of $\pi_{i}$ is the sum of copies of $M_{n}^{0}(\mathbb{Z})$ (matrices of trace 0 ):

$$
\pi_{i}\left(I_{n}+e_{\ell m}\left(\left(t_{j_{1}}-1\right) \cdots\left(t_{j_{i}}-1\right)\right)\right)=\left(0, \ldots, 0, e_{\ell m}(1), 0, \ldots, 0\right)
$$

occurring in the summand corresponding to the monomial $\left(t_{j_{1}}-1\right) \cdots\left(t_{j_{i}}-1\right)$ for $\ell \neq m$. Also, we can hit $e_{\ell, \ell}(1)-e_{\ell+1, \ell+1}(1)$ since

$$
U=\left[\begin{array}{ccccccc}
1 & & & & & & \\
& \ddots & & & & & \\
& & 1 & 1+\left(t_{j_{1}}-1\right) \cdots\left(t_{j_{i}}-1\right) & -\left(t_{j_{1}}-1\right) \cdots\left(t_{j_{i}}-1\right) & & \\
& & \left(t_{j_{1}}-1\right) \cdots\left(t_{j_{i}}-1\right) & 1-\left(t_{j_{1}}-1\right) \cdots\left(t_{j_{i}}-1\right) & 1 & & \\
& & & & & \ddots & 1
\end{array}\right] \in K_{n}^{i}
$$


and $\pi_{i}(U)=e_{\ell, \ell}(1)-e_{\ell+1, \ell+1}(1)+e_{\ell+1, \ell}(1)-e_{\ell, \ell+1}(1)$.

\section{The Main Result}

Let us now investigate the map $\Phi^{i}: \Gamma^{i} F_{n-1} / \Gamma^{i+1} F_{n-1} \rightarrow K_{n}^{i} / K_{n}^{i+1}$. When we refer to a "factor" we mean the copy of $M_{n}(\mathbb{Z})$ or $M_{n}^{0}(\mathbb{Z})$ in $K_{n}^{i} / K_{n}^{i+1}$ corresponding to a certain monomial $\left(t_{j_{1}}-1\right) \cdots\left(t_{j_{i}}-1\right)$; we will abbreviate this monomial to $t_{j_{1}} \cdots t_{j_{i}}$.

Now, we have

$$
\begin{array}{cc}
\Phi^{1}\left(x_{r}\right)=\left(e_{r r}(1)+e_{n r}(-1),\right. & \left.e_{r n}(-1)+e_{n n}(1)\right) \\
t_{n} & t_{r}
\end{array}
$$

where the monomial under an entry indicates the factor in which it lies. For $1 \leq$ $r \leq n-1$, these elements are clearly linearly independent in $K_{n} / K_{n}^{2}=M_{n}(\mathbb{Z})$, and so $\Phi^{1}$ is injective.

On the second level we have, for $r>s$,

$$
\Phi^{2}\left(\left[x_{r}, x_{s}\right]\right)=\left(e_{s r}(-1)+e_{n r}(1), \quad e_{r s}(1)+e_{n s}(-1), \quad e_{r n}(-1)+e_{s n}(1)\right)
$$

and these are clearly linearly independent in $K_{n}^{2} / K_{n}^{3}$. So $\Phi^{2}$ is injective as well.

To calculate $\Phi^{3}$, we must order the bases of $F_{n-1} / \Gamma^{2} F_{n-1}$ and $\Gamma^{2} F_{n-1} / \Gamma^{3} F_{n-1}$. Use the obvious order on the first level: $x_{n-1}>x_{n-2}>\cdots>x_{1}$. On the second level, use

$\left[x_{n-1}, x_{n-2}\right]>\cdots>\left[x_{n-1}, x_{1}\right]>\left[x_{n-2}, x_{n-3}\right]>\cdots>\left[x_{3}, x_{2}\right]>\left[x_{3}, x_{1}\right]>\left[x_{2}, x_{1}\right]$.

Then a basis of $\Gamma^{3} F_{n-1} / \Gamma^{4} F_{n-1}$ is the set

$$
\left[\left[x_{r}, x_{s}\right], x_{u}\right] \quad r>s, u \geq s .
$$

We have the following formula for $\Phi^{3}\left(\left[\left[x_{r}, x_{s}\right], x_{u}\right]\right)$ :

\begin{tabular}{c|l} 
factor & element \\
\hline$t_{s} t_{n}^{2}$ & $e_{s r}\left(\delta_{u s}\right)+e_{s r}\left(-\delta_{u r}\right)+e_{n r}\left(-\delta_{u s}\right)+e_{n r}\left(\delta_{u r}\right)$ \\
$t_{s} t_{u} t_{n}$ & $e_{s n}\left(\delta_{r u}\right)+e_{u r}(1)+e_{n n}\left(-\delta_{r u}\right)+e_{n r}(-1)$ \\
$t_{r} t_{n}^{2}$ & $e_{r s}\left(\delta_{u s}\right)+e_{r s}\left(-\delta_{u r}\right)+e_{n s}\left(\delta_{u r}\right)+e_{n s}\left(-\delta_{u s}\right)$ \\
$t_{r} t_{u} t_{n}$ & $e_{r n}\left(-\delta_{u s}\right)+e_{u s}(-1)+e_{n n}\left(\delta_{u s}\right)+e_{n s}(1)$ \\
$t_{s} t_{r} t_{n}$ & $e_{r n}\left(\delta_{u r}\right)+e_{r u}(1)+e_{n n}\left(-\delta_{u r}\right)+e_{s n}\left(-\delta_{u s}\right)+e_{s u}(-1)+e_{n n}\left(\delta_{u s}\right)$ \\
$t_{r} t_{s} t_{u}$ & $e_{r n}(-1)+e_{s n}(1)$
\end{tabular}

Write $c_{r s u}$ for $\Phi^{3}\left(\left[\left[x_{r}, x_{s}\right], x_{u}\right]\right)$.

Proposition 3.1. $\left\{c_{r s u}: r>s, u \geq s\right\}$ is a linearly independent set in $K_{n}^{3} / K_{n}^{4}$.

Proof. Suppose

$$
\sum m_{r s u} c_{r s u}=0
$$

for some $m_{r s u} \in \mathbb{Z}$. If $s=u$, the factor $t_{s}^{2} t_{r}=t_{r} t_{s} t_{u}$ comes into play and does not occur in any other $c_{r s u}$. So $m_{r s u}=0$ in this case. Similarly if $r=u$, the factor $t_{r}^{2} t_{s}=t_{r} t_{s} t_{u}$ comes into play and does not occur in any other $c_{r s u}$ and so $m_{r s u}=0$ here as well.

Thus, we may assume we have $\sum m_{r s u} c_{r s u}=0$ where each of the $c_{r s u}$ has distinct $r, s, u$. For a given fixed $r, s, u$, the factor $t_{r} t_{s} t_{u}$ occurs exactly twice - in $c_{r s u}$ and $c_{u s r}$. The corresponding elements are $e_{r n}(-1)+e_{s n}(1)$ and $e_{u n}(-1)+e_{s n}(1)$, 
respectively. As $r \neq u$, these are linearly independent in $M_{n}(\mathbb{Z})$, and so we must have $m_{r s u}=m_{u s r}=0$ in this case as well.

Now let's look at $\Phi^{4}$. A basis of $\Gamma^{4} F_{n-1} / \Gamma^{5} F_{n-1}$ consists of the elements

$$
\left[\left[\left[x_{r}, x_{s}\right], x_{u}\right], x_{v}\right] \quad r>s, u \geq s, v \geq u
$$

and

$$
\left[\left[x_{r}, x_{s}\right],\left[x_{u}, x_{v}\right]\right] \quad r>s, u>v, r \geq u
$$

(and in addition, if $r=u$, then $s>v$ ). Denote the image of an element above under $\Phi^{4}$ by $c_{r s u v}$. Note that the order $r, s, u, v$ uniquely determines which of the elements we have, as no sequence from the first type of basis element can occur as

\begin{tabular}{|c|c|}
\hline factor & element \\
\hline$t_{s} t_{n}^{3}$ & $\begin{array}{l}e_{s v}\left(\delta_{u s} \delta_{v r}\right)+e_{v r}\left(-\delta_{v s}\right)+e_{n r}\left(\delta_{s u} \delta_{v s}\right)+e_{s v}\left(-\delta_{u r} \delta_{v r}\right) \\
+e_{v r}\left(\delta_{u r} \delta_{v s}\right)+e_{n r}\left(-\delta_{u r} \delta_{v s}\right)+e_{n v}\left(-\delta_{u s} \delta_{v r}\right)+e_{n v}\left(-\delta_{u r} \delta_{v r}\right)\end{array}$ \\
\hline$t_{s} t_{v} t_{n}^{2}$ & $\begin{array}{l}e_{s n}\left(-\delta_{u s} \delta_{r v}\right)+e_{s n}\left(\delta_{u r} \delta_{r v}\right)+e_{v r}\left(-\delta_{u s}\right)+e_{n n}\left(\delta_{u s} \delta_{r v}\right) \\
+e_{n r}\left(\delta_{u s}\right)+e_{v r}\left(\delta_{u r}\right)+e_{n n}\left(-\delta_{u r} \delta_{r v}\right)+e_{n r}\left(-\delta_{u r}\right) \\
\end{array}$ \\
\hline$t_{u} t_{s} t_{n}^{2}$ & $\begin{array}{l}e_{v n}\left(-\delta_{r u} \delta_{v s}\right)+e_{s v}\left(-\delta_{r u}\right)+e_{n n}\left(\delta_{r u} \delta_{s v}\right)+e_{u v}\left(\delta_{r v}\right) \\
+e_{v r}\left(-\delta_{u v}\right)+e_{n r}\left(\delta_{u v}\right)+e_{n v}\left(\delta_{r u}\right)+e_{n v}\left(-\delta_{r v}\right)\end{array}$ \\
\hline$t_{u} t_{s} t_{v} t_{n}$ & $\begin{array}{l}e_{s n}\left(\delta_{r u}\right)+e_{u n}\left(-\delta_{r v}\right)+e_{v n}\left(-\delta_{r u}\right) \\
+e_{v r}(-1)+e_{n n}\left(\delta_{r v}\right)+e_{n r}(1) \\
\end{array}$ \\
\hline$t_{r} t_{n}^{3}$ & $\begin{array}{l}e_{r v}\left(\delta_{u s} \delta_{v s}\right)+e_{v s}\left(-\delta_{u s} \delta_{v r}\right)+e_{n s}\left(\delta_{u s} \delta_{v r}\right)+e_{r v}\left(-\delta_{u r} \delta_{v s}\right) \\
+e_{v s}\left(\delta_{u r} \delta_{v r}\right)+e_{n s}\left(\delta_{u r} \delta_{v r}\right)+e_{n v}\left(\delta_{u r} \delta_{s v}\right)+e_{n v}\left(-\delta_{u s} \delta_{v s}\right) \\
\end{array}$ \\
\hline$t_{r} t_{v} t_{n}^{2}$ & $\begin{array}{l}e_{r n}\left(-\delta_{u s} \delta_{v s}\right)+e_{r n}\left(\delta_{u r} \delta_{v s}\right)+e_{v s}\left(\delta_{u r}\right)+e_{n n}\left(-\delta_{u r} \delta_{v s}\right) \\
+e_{n s}\left(-\delta_{u r}\right)+e_{v s}\left(-\delta_{u s}\right)+e_{n n}\left(\delta_{u s} \delta_{v s}\right)+e_{n s}\left(\delta_{u s}\right) \\
\end{array}$ \\
\hline$t_{r} t_{u} t_{n}^{2}$ & $\begin{array}{l}e_{v n}\left(\delta_{u s} \delta_{v r}\right)+e_{r v}\left(\delta_{u s}\right)+e_{n n}\left(-\delta_{u s} \delta_{v r}\right)+e_{u v}\left(-\delta_{v s}\right) \\
+e_{v s}\left(\delta_{u v}\right)+e_{n s}\left(-\delta_{u v}\right)+e_{n v}\left(-\delta_{u s}\right)+e_{n v}\left(\delta_{v s}\right) \\
\end{array}$ \\
\hline$t_{r} t_{u} t_{v} t_{n}$ & $\begin{array}{l}e_{r n}\left(-\delta_{u s}\right)+e_{u n}\left(\delta_{v s}\right)+e_{v n}\left(\delta_{u s}\right) \\
+e_{v s}(1)+e_{n n}\left(-\delta_{v s}\right)+e_{n s}(-1) \\
\end{array}$ \\
\hline$t_{r} t_{s} t_{n}^{2}$ & $\begin{array}{l}e_{v n}\left(-\delta_{u r} \delta_{v r}\right)+e_{r v}\left(-\delta_{u r}\right)+e_{n n}\left(\delta_{u r} \delta_{v r}\right)+e_{r v}\left(\delta_{u v}\right)+e_{v u}\left(-\delta_{v r}\right) \\
+e_{n u}\left(\delta_{v r}\right)+e_{n v}\left(\delta_{u r}\right)+e_{v n}\left(\delta_{u s} \delta_{v s}\right)+e_{s v}\left(\delta_{u s}\right)+e_{n n}\left(-\delta_{u s} \delta_{v s}\right) \\
+e_{s v}(-1)+e_{v u}\left(\delta_{v s}\right)+e_{n u}\left(-\delta_{v s}\right)+e_{n u}\left(-\delta_{v s}\right)+e_{n v}\left(-\delta_{v s}\right)\end{array}$ \\
\hline$t_{r} t_{s} t_{v} t_{n}$ & $\begin{array}{l}e_{r n}\left(\delta_{u r}\right)+e_{r n}\left(-\delta_{u v}\right)+e_{v n}\left(-\delta_{u r}\right) \\
+e_{s n}\left(-\delta_{u s}\right)+e_{s n}\left(\delta_{u v}\right)+e_{v n}\left(\delta_{u s}\right) \\
\end{array}$ \\
\hline$t_{r} t_{s} t_{u} t_{n}$ & $\begin{array}{l}e_{v n}\left(\delta_{r v}\right)+e_{v n}\left(-\delta_{s v}\right)+e_{r v}(1) \\
+e_{n n}\left(-\delta_{r v}\right)+e_{s v}(-1)+e_{n n}\left(\delta_{s v}\right) \\
\end{array}$ \\
\hline$t_{r} t_{s} t_{u} t_{v}$ & $e_{r n}(-1)+e_{s n}(1)$ \\
\hline
\end{tabular}
a sequence from the second type. Under $\Phi^{4}$, the image of $\left[\left[\left[x_{r}, x_{s}\right], x_{u}\right], x_{v}\right]$ is 
and the image of $\left[\left[x_{r}, x_{s}\right],\left[x_{u}, x_{v}\right]\right]$ is

\begin{tabular}{c|l} 
factor & element \\
\hline$t_{s} t_{v} t_{n}^{2}$ & $e_{s u}\left(\delta_{r v}\right)+e_{v r}\left(-\delta_{u s}\right)+e_{n r}\left(\delta_{u s}\right)+e_{n u}\left(-\delta_{r v}\right)$ \\
\hline$t_{s} t_{u} t_{n}^{2}$ & $e_{s v}\left(-\delta_{r u}\right)+e_{u r}\left(\delta_{v s}\right)+e_{n r}\left(-\delta_{v s}\right)+e_{n v}\left(\delta_{r u}\right)$ \\
\hline$t_{s} t_{u} t_{v} t_{n}$ & $e_{s n}\left(\delta_{r u}\right)+e_{s n}\left(\delta_{r v}\right)+e_{u r}(1)$ \\
& $+e_{n n}\left(-\delta_{r u}\right)+e_{v r}(-1)+e_{n n}\left(\delta_{r v}\right)$ \\
\hline$t_{r} t_{v} t_{n}^{2}$ & $e_{r u}\left(-\delta_{s v}\right)+e_{v s}\left(\delta_{r u}\right)+e_{n s}\left(-\delta_{r u}\right)+e_{n u}\left(\delta_{s v}\right)$ \\
\hline$t_{r} t_{u} t_{n}^{2}$ & $e_{r v}\left(\delta_{u s}\right)+e_{u s}\left(-\delta_{r v}\right)+e_{n s}\left(\delta_{r v}\right)+e_{n v}\left(-\delta_{s u}\right)$ \\
\hline$t_{r} t_{u} t_{v} t_{n}$ & $e_{r n}\left(-\delta_{u s}\right)+e_{r n}\left(\delta_{v s}\right)+e_{u s}(-1)$ \\
& $+e_{n n}\left(\delta_{u s}\right)+e_{v s}(1)+e_{n n}\left(-\delta_{v s}\right)$ \\
\hline$t_{r} t_{s} t_{v} t_{n}$ & $e_{v n}\left(-\delta_{r u}\right)+e_{r u}(-1)+e_{n n}\left(\delta_{r u}\right)$ \\
& $+e_{v n}\left(\delta_{s u}\right)+e_{s u}(1)+e_{n n}\left(-\delta_{s u}\right)$ \\
\hline$t_{r} t_{s} t_{u} t_{n}$ & $e_{u n}\left(\delta_{r v}\right)+e_{r v}(1)+e_{n n}\left(-\delta_{r v}\right)$ \\
& $+e_{u n}\left(-\delta_{s v}\right)+e_{s v}(-1)+e_{n n}\left(\delta_{s v}\right)$ \\
\hline$t_{r} t_{s} t_{u} t_{v}$ & 0
\end{tabular}

Proposition 3.2. The elements $\left\{c_{r s u v}\right\}$ are linearly independent in $K_{n}^{4} / K_{n}^{5}$.

Proof. Suppose $\sum m_{\text {rsuv }} c_{\text {rsuv }}=0$ for some $m_{\text {rsuv }} \in \mathbb{Z}$. Consider the case where only 2 of the $r, s, u, v$ are distinct (e.g. $c_{2111}, c_{3133}$, etc.). Then $c_{r s u v}$ contributes to the factor $t_{r} t_{s} t_{u} t_{v}=t_{i}^{k} t_{j}^{\ell}$ where $i$ and $j$ are the distinct indices and $1 \leq k, \ell \leq 3$, $k+\ell=4$. Note that $c_{r s u v}$ is the only contributor to this factor as the choice of $r$ determines the sequence - the only possibilities are $r s r r$, rsss or $r s s r$ (note that no $\left[\left[x_{r}, x_{s}\right],\left[x_{u}, x_{v}\right]\right]$ occur as $r \neq s, u \neq v$ implies that $r=u$ and $s=v$ and so the element is 0$)$. To this factor, $c_{r s u v}$ contributes $e_{r n}(-1)+e_{s n}(1)$. It follows that $m_{\text {rsuv }}=0$ for these elements.

Now suppose that $c_{r s u v}=\Phi\left(\left[\left[\left[x_{r}, x_{s}\right], x_{u}\right], x_{v}\right]\right)$ with $r, s, u, v$ distinct (we shall deal with the double commutators with 4 distinct indices below). Then $c_{r s u v}$ contributes the element $e_{r n}(-1)+e_{s n}(1)$ to the factor $t_{r} t_{s} t_{u} t_{v}$. For fixed $r, s, u, v$, we must have $r>s$ and $s<u<v$ if they are all distinct. So the only contributors to this factor are (1) $c_{r s u v}$, (2) $c_{\text {usrv }}$ or $c_{u s v r}$ (the latter if $v<r$ ), (3) $c_{v s r u}$ or $c_{v s u r}$ (the latter if $\left.u<r\right)$; and they contribute $e_{r n}(-1)+e_{s n}(1), e_{u n}(-1)+e_{s n}(1)$, $e_{v n}(-1)+e_{s n}(1)$ respectively. Since these elements are linearly independent in $M_{n}(\mathbb{Z})$, we must have $m_{\text {rsuv }}=m_{u s r v}=m_{v s r u}=0$.

Next, suppose that $r, s, u, v$ consist of 3 distinct indices. We have $r>s, u \geq s$, and $v \geq u$. There are three cases to consider. Recall that in any case, $r>s$.

Case 1. $s=u$. We may assume (without loss of generality) that $r>v$ and $v>u$. Then we have three possible elements to consider: $c_{r s s v}, c_{v s s r}$, and $c_{r s v s}$ (the latter corresponds to $\left.\left[\left[x_{r}, x_{s}\right],\left[x_{v}, x_{s}\right]\right]\right)$. Here, the factor $t_{s}^{2} t_{v} t_{r}$ receives contributions only from $c_{r s s v}$ and $c_{v s s r}$, the elements being $e_{r n}(-1)+e_{s n}(1)$ and $e_{v n}(-1)+e_{s n}(1)$, respectively. These are linearly independent in $M_{n}(\mathbb{Z})$ and so $m_{r s s v}=m_{v s s r}=0$. Then, in the factor $t_{s}^{2} t_{r} t_{n}$, the only contributors are $c_{r s s v}, c_{v s s r}$ and $c_{r s v s}$ - the latter contributing $e_{r v}(1)+e_{s v}(-1)$, while the former two contribute $e_{r v}(1)+e_{s v}(-1)$ and $e_{v r}(1)+e_{s r}(-1)$. As we've already shown that $m_{r s s v}=m_{v s s r}=0$, and since the only other $c_{r s u v}$ that contribute here only have two distinct indices, we must have $m_{\text {rsvs }}=0$ as well.

Case 2. $u=v$. Then $u>s$. Suppose $r>u$ (the case $r<u$ is Case 3 below). Then the three elements to consider are $c_{\text {rsuu }}, c_{\text {usur }}$ and $c_{\text {ruus }}$. The first two elements 
contribute to the factor $t_{s} t_{u}^{2} t_{r}$ the elements $e_{r n}(-1)+e_{s n}(1)$ and $e_{u n}(-1)+e_{s n}(1)$ respectively and no other $c_{i j k l}$ contributes to this factor. So $m_{r s u u}=m_{u s u r}=0$. Then consider the factor $t_{u}^{2} t_{r} t_{n}$. Here, $c_{\text {ruus }}$ contributes $e_{r u}(1)+e_{s u}(-1)$ and the only other contributors have $m_{i j k l}=0$ already. Thus, $m_{\text {ruus }}=0$, as well.

Case 3. $u=v, u>r$. This is similar to Case 2 .

Finally, consider the $c_{r s u v}=\Phi\left(\left[\left[x_{r}, x_{s}\right],\left[x_{u}, x_{v}\right]\right]\right)$ with $r, s, u, v$ distinct. Then $r>s, u>v, r>u$. As the indices are distinct the only factors contributed to are $t_{s} t_{u} t_{v} t_{n}, t_{r} t_{u} t_{v} t_{n}, t_{r} t_{s} t_{v} t_{n}$, and $t_{r} t_{s} t_{u} t_{n}$ (see the formulas above). For fixed $r, s, u, v$, the only possible $c_{i j k l}$ are given in the following table, along with the elements contributed to each factor.

\begin{tabular}{l|c|c|c|c} 
& $t_{s} t_{u} t_{v} t_{n}$ & $t_{r} t_{u} t_{v} t_{n}$ & $t_{r} t_{s} t_{v} t_{n}$ & $t_{r} t_{s} t_{u} t_{n}$ \\
\hline$c_{r s u v}$ & $e_{u r}(1)+e_{v r}(-1)$ & $e_{u s}(-1)+e_{v s}(1)$ & $e_{r u}(-1)+e_{s u}(1)$ & $e_{r v}(1)+e_{s v}(-1)$ \\
\hline$c_{r u s v}$ & $e_{s r}(1)+e_{v r}(-1)$ & $e_{r s}(-1)+e_{u s}(1)$ & $e_{s u}(-1)+e_{v u}(1)$ & $e_{r v}(1)+e_{u v}(-1)$ \\
\hline$c_{r v s u}$ & $e_{s r}(1)+e_{u r}(-1)$ & $e_{r s}(-1)+e_{v s}(1)$ & $e_{r u}(1)+e_{v u}(-1)$ & $e_{s v}(-1)+e_{u v}(1)$
\end{tabular}

In each factor, we obtain linearly dependent elements, but we must remember that we're scaling the element coming from $c_{\text {rsuv }}$ by $m_{\text {rsuv }}$. Looking at the factor $t_{s} t_{u} t_{v} t_{n}$, we find that $m_{\text {rsuv }}=m_{\text {rvsu }}$ and $m_{\text {rusv }}=-m_{\text {rvsu }}$. But then looking at the factor $t_{r} t_{u} t_{v} t_{n}$, we find $m_{r s u v}=-m_{r v s u}$ and $m_{\text {rusv }}=-m_{r v s u}$. Thus, $m_{\text {rsuv }}=m_{\text {rusv }}=m_{\text {rvsu }}=0$.

This completes the proof.

Corollary 3.3. The kernel of $g_{n}$ is contained in $\Gamma^{5} F_{n-1}$.

It is possible to sharpen Corollary 3.3 to obtain the main result.

Theorem 3.4. The kernel of $g_{n}$ is contained in $\left[\Gamma^{3} F_{n-1}, \Gamma^{2} F_{n-1}\right]$.

Proof. For simplicity, denote the group $\Gamma^{i} F_{n-1}$ by $\Gamma^{i}$. By Corollary 3.3 and by $[8$, we have $\operatorname{ker}\left(g_{n}\right) \subseteq \Gamma^{5} \cap\left[\Gamma^{2}, \Gamma^{2}\right]$. We claim that the latter group equals $\left[\Gamma^{3}, \Gamma^{2}\right]$. The main theorem in [6] implies that

$$
\Gamma^{5} \cap\left[\Gamma^{2}, \Gamma^{2}\right]=I_{\Gamma^{2}}\left(\left[\Gamma^{3}, \Gamma^{2}\right]\right),
$$

where $I_{R}(S)$ is the isolator of $S$ in $R$. (Recall that the isolator of $S$ in $R$ is the set $I_{R}(S)=\left\{x \in R: x^{n} \in S\right.$ for some $\left.n\right\}$.) To see that this latter group is simply $\left[\Gamma^{3}, \Gamma^{2}\right]$, it suffices to show that the quotient group $\Gamma^{2} /\left[\Gamma^{3}, \Gamma^{2}\right]$ is torsion-free. Consider the short exact sequence

$$
1 \longrightarrow \frac{\left[\Gamma^{2}, \Gamma^{2}\right]}{\left[\Gamma^{3}, \Gamma^{2}\right]} \longrightarrow \frac{\Gamma^{2}}{\left[\Gamma^{3}, \Gamma^{2}\right]} \longrightarrow \frac{\Gamma^{2}}{\left[\Gamma^{2}, \Gamma^{2}\right]} \longrightarrow 1 .
$$

Since $\Gamma^{2}$ is a free group, the group $\Gamma^{2} /\left[\Gamma^{2}, \Gamma^{2}\right]$ is free abelian. But, by Theorem 6 of [5], the group $\left[\Gamma^{2}, \Gamma^{2}\right] /\left[\Gamma^{3}, \Gamma^{2}\right]$ is also free abelian. It follows that $\Gamma^{2} /\left[\Gamma^{3}, \Gamma^{2}\right]$ is torsion-free. This completes the proof.

The methods used above allow us to prove the following result.

Theorem 3.5. For $s \geq 5, \operatorname{ker}\left(g_{n}\right) \cap \Gamma^{s} \subseteq\left[\Gamma^{s-2}, \Gamma^{2}\right] \cdot \Gamma^{s+1}$.

Proof. Note that any basic commutator is given by a unique list of integers corresponding to the $x_{j}$ that occur in the commutator. For example, $\left[\left[x_{3}, x_{2}\right],\left[x_{3}, x_{1}\right]\right]$ yields the list $3,2,3,1$. We therefore may denote a basic commutator of weight $s$ by $x_{\ell_{1} \ell_{2} \cdots \ell_{s}}$ without confusion. Denote the element $\Phi^{s}\left(x_{\ell_{1} \ell_{2} \cdots \ell_{s}}\right)$ by $c_{\ell_{1} \ell_{2} \cdots \ell_{s}}$. Note 
that all the basic commutators in $\Gamma^{s} / \Gamma^{s+1}$ lie in $\left[\Gamma^{s-2}, \Gamma^{2}\right]$, except for the various $\left[c_{u}, x_{j}\right]$. Moreover, any element of the latter form must be an $s$-fold commutator:

$$
x_{\ell_{1} \ell_{2} \cdots \ell_{s}}=\left[\cdots\left[\left[\left[x_{\ell_{1}}, x_{\ell_{2}}\right], x_{\ell_{3}}\right], \cdots\right], x_{\ell_{s}}\right] .
$$

To prove the theorem, it suffices to show that if we have a dependency relation

$$
\sum m_{\ell_{1} \ell_{2} \cdots \ell_{s}} c_{\ell_{1} \ell_{2} \cdots \ell_{s}}=0
$$

where the $m_{\ell_{1} \ldots \ell_{s}} \in \mathbb{Z}$, then we have $m_{\ell_{1} \ldots \ell_{s}}=0$ whenever $x_{\ell_{1} \ldots \ell_{s}}$ is an $s$-fold commutator. This will show that the $\left[c_{u}, x_{j}\right]$ inject into $K_{n}^{s} / K_{n}^{s+1}$ and hence that the intersection of the kernel of $g_{n}$ with $\Gamma^{s}$ lies in $\left[\Gamma^{s-2}, \Gamma^{2}\right] \cdot \Gamma^{s+1}$.

Observe that in the case of an $s$-fold commutator, the element $c_{\ell_{1} \cdots \ell_{s}}$ contributes the element $e_{\ell_{1}, n}(-1)+e_{\ell_{2}, n}(1)$ to the factor $t_{\ell_{1}} t_{\ell_{2}} \cdots t_{\ell_{s}}$ (this is easily proved by induction using the formulas given above for the $c_{j}$ and $c_{r s}$ ). Suppose the $\ell_{1}, \ell_{2}, \ldots, \ell_{s}$ consist of $i$ distinct indices, say $r_{1}=\ell_{1}, r_{2}=\ell_{2}$, and $r_{3}, \ldots, r_{i}$. We have $r_{1}>r_{2}$ and $r_{2}<r_{3}<\cdots<r_{i}$. We have several contributors to the factor $t_{\ell_{1}} t_{\ell_{2}} \cdots t_{\ell_{s}}=t_{r_{1}}^{a_{1}} t_{r_{2}}^{a_{2}} \cdots t_{r_{i}}^{a_{i}}$ (here $a_{k}$ is the number of times $r_{k}$ occurs). Let us abbreviate notation and write $c_{r_{1} r_{2} \cdots r_{i}}$ for $c_{\ell_{1} \cdots \ell_{s}}$. Certain permutations of the $\ell_{j}$ yield $s$-fold basic commutators; each of these contributes to the factor $t_{r_{1}}^{a_{1}} t_{r_{2}}^{a_{2}} \cdots t_{r_{i}}^{a_{i}}$ under consideration. We must show that the resulting contributions are linearly independent. Note that the only contributors to this factor are $s$-fold commutators - if $c=\left[c_{u}, c_{v}\right]$ where $w\left(c_{v}\right) \geq 2$, then every factor to which $c$ contributes contains a power of $t_{n}$ (see the formulas above). Thus, we may detect any dependency relation among the $c_{\ell_{1}, \ldots, \ell_{s}}$ by considering only the factor $t_{\ell_{1}} \cdots t_{\ell_{s}}$.

Now, for some $q$ with $3 \leq q \leq i$ we must have $r_{q}<r_{1}<r_{q+1}$. Then we get the following contributions to the factor $t_{r_{1}}^{a_{1}} t_{r_{2}}^{a_{2}} \cdots t_{r_{i}}^{a_{i}}$ :

\begin{tabular}{c|c} 
sequence & element \\
\hline$r_{1}, r_{2}, \ldots, r_{i}$ & $e_{r_{1}, n}(-1)+e_{r_{2}, n}(1)$ \\
$r_{q}, r_{2}, \ldots, r_{q-1}, r_{1}, r_{q+1}, \ldots, r_{i}$ & $e_{r_{q}, n}(-1)+e_{r_{2}, n}(1)$ \\
$r_{q+1}, r_{2}, \ldots, r_{q}, r_{1}, r_{q+2}, \cdots, r_{i}$ & $e_{r_{q+1}, n}(-1)+e_{r_{2}, n}(1)$ \\
$r_{q+2}, r_{2}, \ldots, r_{q}, r_{1}, r_{q+1}, r_{q+3}, \ldots, r_{i}$ & $e_{r_{q+2}, n}(-1)+e_{r_{2}, n}(1)$ \\
$\vdots$ & $\vdots$ \\
$r_{i}, r_{2}, \ldots, r_{q}, r_{1}, r_{q+1}, \ldots, r_{i-1}$ & $e_{r_{i}, n}(-1)+e_{r_{2}, n}(1)$
\end{tabular}

Since $r_{1}, r_{2}, \ldots, r_{i}$ are distinct, the elements in the second column are linearly independent in $M_{n}(\mathbb{Z})$ (as $\left.i<n\right)$ and so each of the corresponding coefficients satisfies $m_{\ell_{1} \cdots \ell_{s}}=0$. This completes the proof.

\section{BREAKdown}

The method used in Section 3 breaks down at the fifth level, however. Indeed, if $n=4$, the kernel of $\Phi^{5}$ is rather large. For example, we have $c_{21131}=c_{31121}$. This has the interpretation that the degree 5 part of the polynomials in the Gassner matrices of

and

$$
\left[\left[\left[A_{24}, A_{14}\right], A_{14}\right],\left[A_{34}, A_{14}\right]\right]
$$

$$
\text { [[[ } \left.\left.\left.A_{34}, A_{14}\right], A_{14}\right],\left[A_{24}, A_{14}\right]\right]
$$

are the same. The matrices are not the same, however, and a computer search by the author based on the relations in the kernel of $\Phi^{5}$ has not turned up any elements in the kernel of $G_{4}$. 
Note, however, that the failure of the method does not imply that $g_{n}$ is not injective. Really, one needs to consider the quotients $\Gamma^{i} g_{n}\left(F_{n-1}\right) / \Gamma^{i+1} g_{n}\left(F_{n-1}\right)$ rather than the classes of the various elements in $\Gamma^{i} g_{n}\left(F_{n-1}\right)$ modulo the subgroup $K_{n}^{i+1}$. This seems to be rather intractable, however, given the ranks of the various $\Gamma^{i} F_{n-1} / \Gamma^{i+1} F_{n-1}$ (for example, $\Gamma^{5} F_{3} / \Gamma^{6} F_{3}$ has rank 116 ).

\section{REFERENCES}

[1] M. Abdulrahim, The reduced Gassner representation restricted to a normal free subgroup of the pure braid group, Arch. Math. (Basel) 69 (1997), 8-12.

[2] J. Birman, Braids, links and mapping class groups, Annals of Math. Studies no. 82, 1975.

[3] F. Cohen, S. Prassidis, On injective homomorphisms for pure braid groups, and associated Lie algebras, preprint (2004).

[4] M. Hall, The theory of groups, The Macmillan Company, New York, 1959.

[5] T. Hurley, Some properties of certain relatively free groups, Proc. Amer. Math. Soc. 47 (1975), 317-322.

[6] T. Hurley, Identifications in a free group, J. Pure Appl. Algebra 48 (1987), 249-261.

[7] K. Knudson, Relative completions of linear groups over $\mathbb{Z}[t]$ and $\mathbb{Z}\left[t, t^{-1}\right]$, Trans. Amer. Math. Soc. 352 (2000), 2205-2216.

[8] S. Lipschutz, On a finite matrix representation of the braid group, Arch. Math. (Basel) 12 (1961), 7-12.

Department of Mathematics and Statistics, Mississippi State University, Mississippi State, MS 39762

E-mail address: knudson@math.msstate.edu 